OPEN ACCESS

Edited by: Jacob Dean,

Southern Utah University,

United States

Reviewed by:

Takayuki Ebata,

National Chiao Tung University, Taiwan

Ryan Pensack,

Boise State University, United States

Christian W. Mueller,

French-German Research Institute of

Saint-Louis, France

*Correspondence:

Vasilios G. Stavros

v.stavros@warwick.ac.uk

Specialty section:

This article was submitted to

Physical Chemistry and Chemical

Physics,

a section of the journal

Frontiers in Chemistry

Received: 18 June 2020

Accepted: 26 August 2020

Published: 25 September 2020

Citation:

Whittock AL, Turner MAP, Coxon DJL,

Woolley JM, Horbury MD and

Stavros VG (2020) Reinvestigating the

Photoprotection Properties of a

Mycosporine Amino Acid Motif.

Front. Chem. 8:574038.

doi: 10.3389/fchem.2020.574038

\section{Reinvestigating the Photoprotection Properties of a Mycosporine Amino Acid Motif}

\author{
Abigail L. Whittock ${ }^{1,2}$, Matthew A. P. Turner ${ }^{2,3,4}$, Daniel J. L. Coxon ${ }^{2,4,5}$, Jack M. Woolley ${ }^{2}$, \\ Michael D. Horbury ${ }^{6}$ and Vasilios G. Stavros ${ }^{2 *}$ \\ ${ }^{1}$ Analytical Science Centre for Doctoral Training, Senate House, University of Warwick, Coventry, United Kingdom, \\ ${ }^{2}$ Department of Chemistry, University of Warwick, Coventry, United Kingdom, ${ }^{3}$ Molecular Analytical Science Centre for \\ Doctoral Training, Senate House, University of Warwick, Coventry, United Kingdom, ${ }^{4}$ Department of Physics, University of \\ Warwick, Coventry, United Kingdom, ${ }^{5}$ Diamond Science and Technology Centre for Doctoral Training, University of Warwick, \\ Coventry, United Kingdom, ${ }^{6}$ School of Electronic and Electrical Engineering, University of Leeds, Leeds, United Kingdom
}

With the growing concern regarding commercially available ultraviolet (UV) filters damaging the environment, there is an urgent need to discover new UV filters. A family of molecules called mycosporines and mycosporine-like amino acids (referred to as MAAs collectively) are synthesized by cyanobacteria, fungi and algae and act as the natural UV filters for these organisms. Mycosporines are formed of a cyclohexenone core structure while mycosporine-like amino acids are formed of a cyclohexenimine core structure. To better understand the photoprotection properties of MAAs, we implement a bottom-up approach by first studying a simple analog of an MAA, 3-aminocyclohex-2-en-1-one (ACyO). Previous experimental studies on ACyO using transient electronic absorption spectroscopy (TEAS) suggest that upon photoexcitation, ACyO becomes trapped in the minimum of an $S_{1}$ state, which persists for extended time delays (>2.5 ns). However, these studies were unable to establish the extent of electronic ground state recovery of ACyO within 2.5 ns due to experimental constraints. In the present studies, we have implemented transient vibrational absorption spectroscopy (as well as complementary TEAS) with Fourier transform infrared spectroscopy and density functional theory to establish the extent of electronic ground state recovery of $\mathbf{A C y O}$ within this time window. We show that by $1.8 \mathrm{~ns}$, there is $>75 \%$ electronic ground state recovery of $\mathbf{A C y O}$, with the remaining percentage likely persisting in the electronic excited state. Long-term irradiation studies on $\mathbf{A C y O}$ have shown that a small percentage degrades after $2 \mathrm{~h}$ of irradiation, plausibly due to some of the aforementioned trapped $\mathbf{A C y O}$ going on to form a photoproduct. Collectively, these studies imply that a base building block of MAAs already displays characteristics of an effective UV filter.

Keywords: photoprotection, photostability, ultrafast, spectroscopy, mycosporine 


\section{INTRODUCTION}

Ultraviolet (UV) radiation, in particular UVA (315-400 nm) and UVB $(280-315 \mathrm{~nm})$, that reaches the Earth's surface has both positive and negative effects on living organisms (Holick, 2004; Lucas et al., 2006; Humble, 2010). Consequently, nature has developed its own photoprotection to safeguard from harmful DNA damage. In humans, this natural photoprotection is achieved by producing melanin pigments that can absorb UVA and UVB radiation before it reaches DNA (Kollias et al., 1991). However, as melanin production is a delayed process and does not absorb all UVA and UVB radiation that reaches the skin, a more immediate form of protection is required, i.e., sunscreens (Eller and Gilchrest, 2000; Brenner and Hearing, 2008; Wang and Lim, 2016). Some UV filters found in sunscreens have been linked to the cause of damaging environmental effects as well as adverse dermatological effects (Bryden et al., 2006; Danovaro et al., 2008; Downs et al., 2013, 2016; Warshaw et al., 2013; Schaap and Slijkerman, 2018). As a result, there is an increased need for the identification of new UV filters that are less harmful to both the environment and humans. Drawing inspiration from nature offers a promising solution to these negative impacts. One approach is to base candidate UV filters off structures observed in plants and microorganisms (Bandaranayake, 1998; Dean et al., 2014; Baker et al., 2016, 2018; Horbury et al., 2017, 2018, 2019; Luo et al., 2017; Zhao et al., 2019a,b).

Cyanobacteria, fungi, macro- and microalgae all synthesize a family of molecules termed mycosporines and mycosporine-like amino acids (Sinha et al., 2007; Balskus and Walsh, 2010). In the literature, mycosporines and mycosporine-like amino acids are used synonymously. However, mycosporines are a family of molecules comprised of a cyclohexenone core and mycosporinelike amino acids are comprised of a cyclohexenimine core (herein both mycosporines and mycosporine-like amino acids will be termed MAAs) (Gao and Garcia-Pichel, 2011). MAAs present a strong absorbance band in the UVA and UVB, and their high photostability makes them highly desirable as potential UV filters (Bandaranayake, 1998; Conde et al., 2000, 2007; Sinha et al., 2000; Moliné et al., 2011; Rastogi and Incharoensakdi, 2014). As the extraction from natural sources and the synthetic preparation of natural MAAs produces small quantities, some work has been carried out on synthetic MAA motifs as an alternative; these molecules have also demonstrated promising levels of photostability thus far (White et al., 1989, 1995; Bandaranayake, 1998; Losantos et al., 2017, 2019; Woolley et al., 2018). Complementary studies, implementing computational and ultrafast spectroscopic techniques have been conducted on various MAA motifs in an effort to guide future UV filter design through knowledge of the photoprotection mechanisms

\footnotetext{
Abbreviations: UV, ultraviolet; MAA, mycosporine-like amino acid; ACyO, 3aminocyclohex-2-en-1-one; TEAS, transient electronic absorption spectroscopy; CI, conical intersection; ESA, excited state absorption; GSB, ground state bleach; TVAS, transient vibrational absorption spectroscopy; DFT, density functional theory; EtOH, ethanol; DMSO, dimethyl sulfoxide; TDDFT, timedependent density functional theory; FTIR, Fourier transform infrared; TEA, transient electronic absorption; MCT, mercury cadmium telluride; TVA, transient vibrational absorption.
}

(Losantos et al., 2017, 2019; Woolley et al., 2018). Their finding will be briefly outlined here.

Losantos et al. (2017) implemented a CASPT2/CASSCF methodology to evaluate the minimum energy paths of a selection of MAA motifs composed of either a cyclohexenone or a cyclohexenimine core. Their results showed that a cyclohexenone core is potentially a poor scaffold for sunscreen applications, as following photoexcitation to the lowest optically bright $S_{2}$ excited state, a geometric distortion to a nonplanar geometry leads to relaxation to the $S_{1}$ state via a $\mathrm{S}_{2} / \mathrm{S}_{1}$ conical intersection (CI). The electronic excited state population then traverses along the potential energy surface of the $S_{1}$, before becoming trapped in a minimum on the $S_{1}$ potential energy surface. For the cyclohexenimine core, Losantos et al. (2017) proposed that after initial photoexcitation to the lowest optically bright $S_{1}$ excited state, fast relaxation along an out-of-plane geometry distortion leads to an accessible $\mathrm{S}_{1} / \mathrm{S}_{0} \mathrm{CI}$, resulting in efficient repopulation of the electronic ground state. These results by Losantos et al. (2017) are corroborated by computational studies on the natural MAAs palythine and porphyra-334 (Sampedro, 2011; Koizumi et al., 2017; Hatakeyama et al., 2019). Interestingly, a computational study on gadusol (a molecule closely related to MAAs with a cyclohexenone core) found an accessible barrierless $S_{1} / S_{0}$ CI (Losantos et al., 2015). This, in addition to experimental studies on gadusol which reported high levels of photostability and rapid non-radiative decay as the dominant relaxation pathway (Arbeloa et al., 2011), indicates that molecules with a cyclohexenone core do display a number of ideal properties of a UV filter. Therefore, there is still much to learn about these cyclohexenone systems.

Woolley et al. (2018) and Losantos et al. (2019) have conducted transient electronic absorption spectroscopy (TEAS) experiments on MAA motifs to unravel their photoprotection mechanisms. Woolley et al. (2018) investigated two MAA motifs, the one of interest for this paper being 3-aminocyclohex-2en-1-one (ACyO), see inset of Figure 1 for the structure of ACyO. Their TEAS experimental results for ACyO in methanol (polar protic) and acetonitrile (polar aprotic) demonstrated a persistent excited state absorption (ESA), $>2.5 \mathrm{~ns}$, corroborating calculations from Losantos et al. (2017) that (at least a fraction of) ACyO becomes trapped in a minimum of the $S_{1}$. We note here that the calculated barrier to the $\mathrm{S}_{1} / \mathrm{S}_{0} \mathrm{CI}$ is $0.04 \mathrm{eV}$ for $\mathrm{ACyO}$ in the gas-phase (Woolley et al., 2018). Whilst population persisted in the excited state for nanoseconds, no real indication as to the percentage of ACyO that remained in the electronic excited state can be inferred from the TEAS experiment. This is due to TEAS experimental limitations as Woolley et al. (2018) were unable to observe a ground state bleach (GSB) of ACyO. Consequently, they could not establish the extent of electronic ground state recovery which would give a direct measure of the photostability of ACyO. Herein, we propose that UV pump infrared probe transient vibrational absorption spectroscopy (TVAS) can be used to measure the extent of vibrational ground state recovery in the electronic ground state on ultrafast timescales after photoexcitation, thus providing insight into the overall electronic ground state recovery of $\mathrm{ACyO}$. 


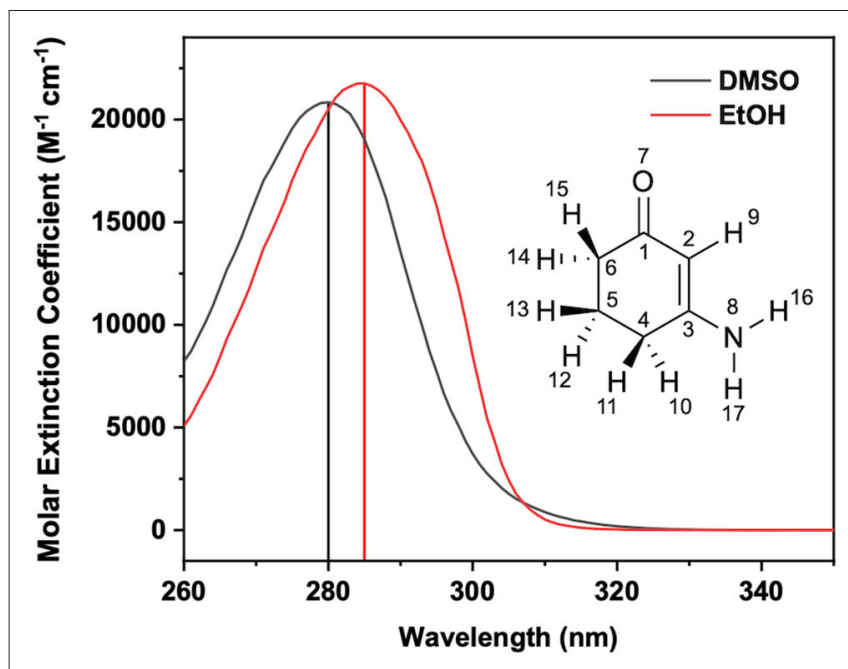

FIGURE 1 | Steady-state UV/visible extinction spectra of ACyO in EtOH (black line) and DMSO (red line). Vertical line corresponds to the absorption maxima of each solution; $285 \mathrm{~nm}$ for $\mathrm{EtOH}$ and $280 \mathrm{~nm}$ for DMSO. Inset is the structure of $\mathbf{A C y O}$ along with its atom numbers.

To better understand the behavior of MAA motifs with a cyclohexenone core, we have performed TVAS, along with complementary TEAS and long-term irradiation studies on ACyO, to study the GSB recovery of its vibrational modes. We build on a study by Sui et al. (2012) who used density functional theory (DFT) to assign the vibrational modes of ACyO in acetonitrile and water; for reasons that will become apparent later, our experiments utilized ethanol (EtOH) and dimethyl sulfoxide (DMSO) as solvents. In order to assign the vibrational modes both in the electronic ground and excited state in $\mathrm{EtOH}$ and DMSO, we have implemented implicitand explicit-solvent DFT and time-dependent DFT (TDDFT) (Casida, 1995) calculations using a similar level of theory to Sui et al. (2012). These high-level calculations were necessary for the identification of any overlapping electronic ground and excited state frequencies that would influence the interpretation of our TVAS data. We report in the present work that TVAS is a powerful technique that can extract GSB recovery quantum yields for the vibrational modes of a molecule on ultrafast timescales by comparing the extent of the bleach recovery with respect to the initial GSB. Previous publications have reported GSB recovery quantum yields, and how such qualitative information provides valuable insight into the efficiency of relaxation pathways (Holm et al., 2003; Rini et al., 2003; Murdock et al., 2014, 2015). For ACyO, the resultant quantum yields in addition to its overall photostability demonstrates promising properties that are required of a UV filter, providing some evidence for the photoprotective capabilities of MAAs with a cyclohexenone core.

\section{MATERIALS AND METHODS}

ACyO was purchased from Alfa Aesar (95\% purity) and solutions of $\sim 40 \mu \mathrm{M}, 4 \mathrm{mM}$, and $50 \mathrm{mM}$ concentrations were made up in the two solvents used in this study. $\mathrm{EtOH}(\geq 99.8 \%)$ and DMSO $(\geq 99.9 \%)$ were purchased from VWR Chemicals and Fisher Scientific, respectively.

\section{Steady-State Spectroscopy}

ACyO samples in EtOH and DMSO of concentrations $\sim 40 \mu \mathrm{M}$ contained in a $1 \mathrm{~cm}$ path length quartz cuvette were irradiated within a solar simulator (91,191-1,000, Oriel Instruments) which has an output power equivalent to the sun at the Earth's surface $\left(\sim 1,000 \mathrm{~W} / \mathrm{m}^{2}\right)$ for $7,200 \mathrm{~s}(120 \mathrm{~min}, 2 \mathrm{~h})$. At various time intervals, UV/visible spectra were recorded using a UV/visible spectrometer (Cary 60, Agilent Technologies). To confirm that the degradation occurring was due to photodegradation, UV/visible spectra of ACyO solutions that had not been irradiated were taken before and after 7,200 s.

Fourier transform infrared (FTIR) spectra were obtained using a FTIR spectrometer (VERTEX 70v, Bruker) under a nitrogen environment to remove vibrational modes associated with atmospheric gases. $50 \mathrm{mM}$ ACyO solutions in EtOH and DMSO were contained within a demountable liquid cell (Harrick Scientific Products Inc.) with a path length of $100 \mu \mathrm{m}$ achieved by inserting $100 \mu \mathrm{m}$ PTFE spacers between two $\mathrm{CaF}_{2}$ windows (front window $1 \mathrm{~mm}$ and back window $2 \mathrm{~mm}$ thickness). The FTIR spectra were recorded over an energy range of 500-4,000 $\mathrm{cm}^{-1}$ with a resolution of $1 \mathrm{~cm}^{-1}$.

\section{Ultrafast Spectroscopy}

TEAS and TVAS measurements were taken at the Warwick Center for Ultrafast Spectroscopy (WCUS: www.go.warwick. ac.uk/fac/sci/wcus). The setup of the TEAS experiment has been previously reported (Woolley et al., 2018), but it will be briefly described here. $4 \mathrm{mM}$ solutions of ACyO in EtOH and DMSO were circulated through a demountable liquid cell with a path length of $100 \mu \mathrm{m}$ set up in the same way as for FTIR spectroscopy described above. The samples were continuously circulated through the cell using a diaphragm pump (SIMDOS 02) to ensure a fresh sample was interacting with each laser shot. $800 \mathrm{~nm}$ pulses $(12 \mathrm{~W}, 1 \mathrm{kHz}, 40 \mathrm{fs})$ were generated by a Ti:Sapphire regenerative amplified laser system (Dual Ascend Pumped Spitfire Ace, Spectra-Physics) seeded by a Mai Tai (Spectra-Physics). The beam was split into four fractions, each fraction having its own recompression grating. One of the four fractions $(3.5 \mathrm{~W})$ was split into two further fractions in order to generate the pump and probe beams required for the TEAS experiments.

The first of the fractions $(2.5 \mathrm{~W})$ seeds an optical parametric amplifier (Topas-Prime with UV-extension, Light Conversion) which allows for a tuneable pump beam wavelength that includes 285 and $280 \mathrm{~nm}$ for photoexciting ACyO in EtOH and DMSO, respectively. These wavelengths were chosen as they correspond to the absorption maximum of each ACyO solution as displayed in Figure 1. The pump beam was set to a power of $\sim 500 \mu \mathrm{W}$ and was focused beyond the sample holder to give a beam diameter of $500 \mu \mathrm{m}$ at the sample holder. The probe beam was generated by focussing $5 \%$ of the remaining $1 \mathrm{~W}$ fundamental $800 \mathrm{~nm}$ beam which had been further attenuated and irised on to a vertically translated $\mathrm{CaF}_{2}$ window ( $2 \mathrm{~mm}$ thickness) to 
generate a white light continuum (320-720 nm). The probe pulse polarization was held at the magic angle $\left(54.7^{\circ}\right)$ with regards to the pump pulse polarization; this is done to avoid dynamical contributions from molecular reorientations. The path length of the $800 \mathrm{~nm}$ beam used to generate the probe beam can be varied to give pump-probe time delays $(\Delta t)$ of -1 ps to 2.5 ns using a gold retroreflector mounted on a motorized optical delay line. Before reaching the sample, the pump beam passes through an optical chopper operating at a repetition rate of $500 \mathrm{~Hz}$, blocking every other pulse of the $1 \mathrm{kHz}$ pulse train. This allows for direct comparison of the signal detected by a fiber-coupled spectrometer (AvaSpec-ULS1650F, Avantes) for the pumped and unpumped samples which is displayed as changes in optical density $(\triangle \mathrm{OD})$ in the resultant transient electronic absorption (TEA) spectra.

Pump power dependency studies were carried out to ensure that $\triangle \mathrm{OD}$ was linearly dependent on photon flux (see Supplementary Figure 1). Chirp correction of the TEA spectra was achieved using the KOALA package (Grubb et al., 2014). Global fitting of the TEA spectra was employed using the software package Glotaran (Mullen and Van Stokkum, 2007; Snellenburg et al., 2012), and a sequential kinetic model $\left(\mathrm{A} \stackrel{\tau_{e 1}}{\longrightarrow} \mathrm{B} \stackrel{\tau_{e 2}}{\longrightarrow} \mathrm{C} \ldots\right)$ was used over the entire spectral region of our probe $(320-720 \mathrm{~nm})$. The evolution associated difference spectra for the time constants of each fit can be found in Supplementary Figure 2. The quality of the fits was assessed through the associated residuals, see Supplementary Figure 2. The instrument response function, accounting for the temporal resolution of our TEAS measurements, was determined by fitting a Gaussian over the time zero artifacts of solventonly scans and taking the full width half-maximum, see Supplementary Figure 3 for the instrument response functions of EtOH and DMSO.

For the TVAS set up, $50 \mathrm{mM}$ ACyO solutions in EtOH and DMSO were circulated through a demountable liquid cell the same way as reported above for the TEAS set-up. The pump beam was set to a higher power of $700 \mu \mathrm{W}$ for the TVAS experiments. The probe beam $(240 \mu \mathrm{W}, 360 \mu \mathrm{m}$ beam diameter for the TVAS experiment was generated by seeding a second optical parametric amplifier (Topas-C, Spectra-Physics) with a second $3.5 \mathrm{~W}$ fraction of the fundamental $800 \mathrm{~nm}$ beam. The generated probe beam allows for a tuneable IR wavelength that includes 6,420 and $6,289 \mathrm{~nm}$ which were the wavelengths used in this experiment for ACyO in EtOH and DMSO, respectively. The path length of the probe beam was varied in a similar way to the TEAS set-up to give $\Delta t$ of -1 ps to $1.8 \mathrm{~ns}$. Atmospheric absorption lines were excluded by purging the probe line with nitrogen gas. Before the probe beam arrived at the sample compartment, it was incident on $\mathrm{CaF}_{2}$ beamsplitter where it was split equally in to reference and probe pulses. The reference pulse did not traverse the sample and was detected for the subtraction of shot-to-shot laser noise. The probe pulse passed through the sample where it was partially absorbed before entering an imaging spectrometer (iHR320, HORIBA Scientific). Once in the spectrometer, the probe was dispersed by a diffraction grating ( $6 \mu \mathrm{m}$ blaze, 100 lines $\mathrm{mm}^{-1}$ resolution) onto a mercury cadmium telluride (MCT) detector array (FPAS-0144, Infrared
Systems Development) which was made up of two 64-pixel linear arrays-one each for the probe and reference pulses. The MCT detector was cooled using liquid nitrogen to eliminate thermal contributions to the signal. Like in the TEAS experiment, the pump beam passes through an optical chopper operating at a repetition rate of $500 \mathrm{~Hz}$, blocking every other pulse of the $1 \mathrm{kHz}$ pulse train. This allows for direct comparison of the signal for the pumped and unpumped samples which is displayed as $\triangle \mathrm{OD}$ in the resultant transient vibrational absorption (TVA) spectra.

We converted between pixel number and wavelength using a mid-IR polystyrene calibration card (Perkin Elmer) as our reference. Similarly to the TEAS, pump power dependency studies were carried out, see Supplementary Figure 4. Only $\sim 100 \mathrm{~cm}^{-1}$ windows were investigated for each solvent environment; this was dictated by the spectral congestion emanating from the solvent and the transition intensity of ACyO vibrational modes. Exponential fits of the GSB features were employed to extract time constants associated with the dynamical processes ACyO undergoes upon photoexcitation.

\section{Computational Methods}

Vibrational frequencies in the electronic ground $\left(\mathrm{S}_{0}\right)$ and excited $\left(S_{1}\right)$ state were predicted for ACyO in both implicitand explicit-solvent environments for $\mathrm{EtOH}$ and DMSO. The NWChem package (Valiev et al., 2010) was used to perform DFT and TDDFT calculations. These were done at the PBE0/cc-pVTZ level of theory (Davidson, 1996; Perdew et al., 1996; Adamo and Barone, 1999). Implicit-solvent calculations were employed using the COSMO solvent model for both EtOH and DMSO which models each solvent's dielectric parameters (Klamt and Schüürmann, 1993; Winget et al., 1999; York and Karplus, 1999). We add that the level of theory described has previously been used for a similar system studied by Turner et al. (2019) which also performed vibrational frequency calculations. Due to discrepancies in the calculated $\mathrm{S}_{0}$ vibrational frequencies and their corresponding vibrational modes, in addition to the fact $\mathrm{EtOH}$ and DMSO interact strongly with ACyO, it was determined that explicit-solvent contributions should be considered. See Supplementary Table 1 and Supplementary Figure 5 for implicit-solvent vibrational frequency results. This high-level of theory has been used so that accurate electronic excited state frequencies are predicted, which in turn provides valuable insight into any overlapping electronic ground and excited state frequencies in the TVAS probe region. As a result, reliable GSB recovery quantum yields can be extracted from the TVA spectra. Although neither implicit- or explicit-solvent results are perfect, the scaling factors required for explicit-solvent (0.997 and 0.982 for EtOH and DMSO, respectively) were significantly closer to 1 than implicitsolvent ( 0.976 for both solvents) indicating improved accuracy for the explicit-solvent calculations. Therefore, the decision was made to report explicit-solvent herein. Further to this, the asymmetry of the $1.8 \mathrm{~ns}$ lineout from our TVA spectra can be explained by the computed $S_{1}$ frequencies determined by EtOH explicit-solvent (see later discussion). On the other hand, the computed $S_{1}$ frequencies for EtOH implicit-solvent do not provide such clarity. Furthermore, regardless of whether an 
implicit- or explicit-solvent model is used for ACyO in DMSO, there are no overlapping $S_{1}$ frequencies with the probed peaks in the TVAS experiment.

Solvent shells were generated through a similar method used by Zuehlsdorff et al. (2017) and Turner et al. (2019). In this, a classical molecular dynamics simulation was performed using the AMBER package (Case et al., 2015). ACyO, optimized in DFT at the level of theory discussed previously, was immersed in a $20 \AA$ cube of explicit-solvent (both EtOH and DMSO). The system was heated over 20 ps in the NVT ensemble wherein the temperature is raised from 0 to $300 \mathrm{~K}$. This, along with all further calculations, was achieved using a Langevin thermostat with a collision frequency of $1 \mathrm{ps}^{-1}$. Following this, a 400 ps pressure equilibration is utilized in the NPT isothermic-isobaric ensemble with the pressure fixed at $1 \mathrm{~atm}$. Next, the system was equilibrated at a constant temperature of $300 \mathrm{~K}$ for 100 ps. Finally, snapshots were generated via a further NVT ensemble run, this time with a fixed temperature of $300 \mathrm{~K}$ for 8 ns. 2000 snapshots were extracted by recording a frame every 4 ps. By saving every tenth snapshot, 200 of these snapshots were exported. Within each snapshot, the solute and hydrogen bonding solvent molecules were extracted by removing any solvent molecules that were further than $1.5 \AA$ from the solute.

As has been previously conducted by Turner et al. (2019), four snapshots were chosen for each solvent. Each snapshot has a different solvent environment and initial structures are shown in Supplementary Table 2. In the case of DMSO, only two solvent molecules were within $1.5 \AA$ of ACyO and hence the four chosen snapshots were selected to ensure that the DMSO molecules were in different orientations in each snapshot (see Supplementary Table 2). For EtOH, a variety of solvent environments over the 200 snapshots were found, the most common being; one $\mathrm{EtOH}$ on the carbonyl oxygen and one $\mathrm{EtOH}$ on the amine hydrogen, two $\mathrm{EtOH}$ on the carbonyl oxygen and one $\mathrm{EtOH}$ on the amine hydrogen, one $\mathrm{EtOH}$ on the carbonyl oxygen and two EtOH on the amine hydrogens and finally two EtOH on the carbonyl oxygen and two EtOH on the amine hydrogens. One of each environment was selected for the calculations in order to evaluate the different solvent environments determined by molecular dynamics (see Supplementary Table 2). Calculations of full solvent shells were not conducted for the present work. This is due to previous findings by Turner et al. (2019) who found that full solvent shell calculations and smaller clusters with only two solvent molecules did not generate notable differences in the calculated vibrational frequencies. Furthermore, such calculations take significant computational expenditure due to the larger number of atoms.

The DFT calculations that were conducted were as follows: initially a geometry optimisation was employed on all the implicit- and explicit-solvent structures stated above followed by an electronic ground state frequency calculation. Vertical excitations were conducted and the results corroborated previous work stating that the first optically bright state is the $S_{2}$ (Sui et al., 2012; Losantos et al., 2017). Following this calculation, electronic excited state geometry relaxation calculations were carried out to determine the relaxed geometry on the $S_{1}$ excited state. The $S_{1}$ was chosen based on previous work that computed the minimum energy path and found that ACyO becomes trapped in the minimum of its $S_{1}$ excited state (Losantos et al., 2017; Woolley et al., 2018). Finally, electronic excited state vibrational frequency calculations were conducted on the $\mathrm{S}_{1}$ relaxed geometry. Only averaged explicit-solvent vibrational frequencies for ACyO in EtOH and DMSO are presented herein, but all individual results for the implicit- and explicit-solvent environments can be found in the Supplementary Material including the optimised geometries and vertical excitation results (see Supplementary Tables 1-5). We also note that all reported frequencies are between 1,500 and $1,750 \mathrm{~cm}^{-1}$ as it is the region of interest for the present work and extensive evaluation of the computed frequencies is outside the scope of this work.

Scaling factors were applied to the calculated $S_{0}$ and $S_{1}$ frequencies and were calculated by using one experimental peak as a reference so that the calculated frequency exactly matches the reference experimental peak. This method is common practice and has been employed in a similar study by Turner et al. (2019) and Grieco et al. (2018). The chosen $\mathrm{S}_{0}$ reference peaks were 1,559 and $1,577 \mathrm{~cm}^{-1}$ for ACyO in EtOH and DMSO, respectively, and were chosen because they were the strongest experimental peaks observed between 1,500 and $1,750 \mathrm{~cm}^{-1}$. As there were no clear experimental $S_{1}$ frequencies obtained in this study, we tentatively applied the same scaling factor to the $S_{1}$ frequency calculation as was applied to the $S_{0}$ frequency calculation for both solvent environments; a similar approach was implemented by Baker et al. (2015). The resultant scaling factors for the averaged explicit-solvents snapshots of ACyO in EtOH and DMSO were 0.997 and 0.982 , respectively.

\section{RESULTS AND DISCUSSION}

We first take a look at the TEA spectra acquired for ACyO in EtOH and DMSO which are displayed as false color maps in Figure 2; for lineouts of the TEA spectra see Supplementary Figure 6. The choice to use EtOH and DMSO as solvents was firstly to demonstrate dynamics in different solvent types (protic and aprotic), and secondly because these solvents displayed clear spectral windows in the region of interest for our FTIR and TVAS experiments. We note here that our TEA spectra strongly correlate with the findings of Woolley et al. (2018); the ACyO excited-state dynamics in EtOH are very similar to those observed in methanol (as might be expected; both polar and protic), and the ACyO excited state dynamics in DMSO are also very similar to those observed in acetonitrile (both polar and aprotic). Hence only a brief discussion is required here. Starting with $\mathbf{A C y O}$ in EtOH shown in Figure 2A, we observe a negative feature at early pump-probe time delays $(\Delta t<0.2 \mathrm{ps})$ identified as multiphoton initiated dynamics (see Supplementary Figure 1), followed by an intense ESA centered at $\sim 330 \mathrm{~nm}$ which blue-shifts out of the probe window and mostly decays by $\sim 5$ ps. A second ESA of less intensity grows in within 5 ps centered at $\sim 400 \mathrm{~nm}$ and persists to the final time delay of our experiment $(\Delta t=2.5 \mathrm{~ns})$, see Supplementary Figure 6A for lineouts. We now take a look at 
A

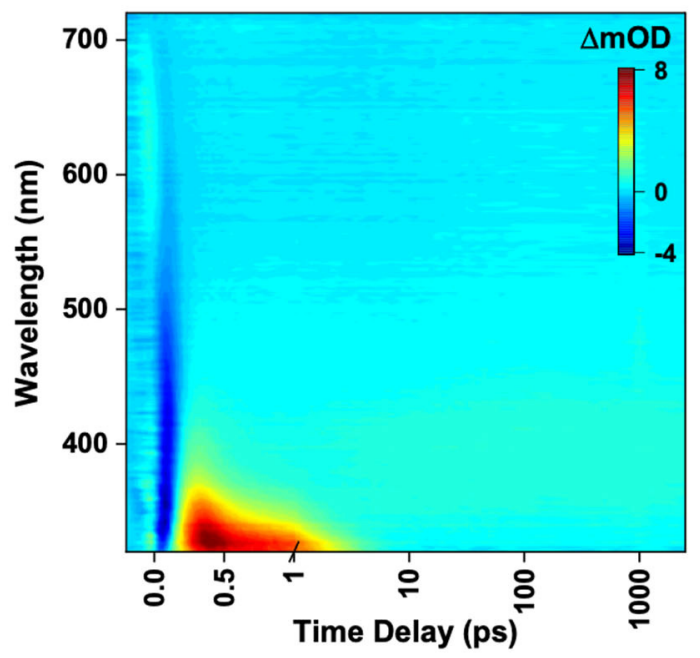

B

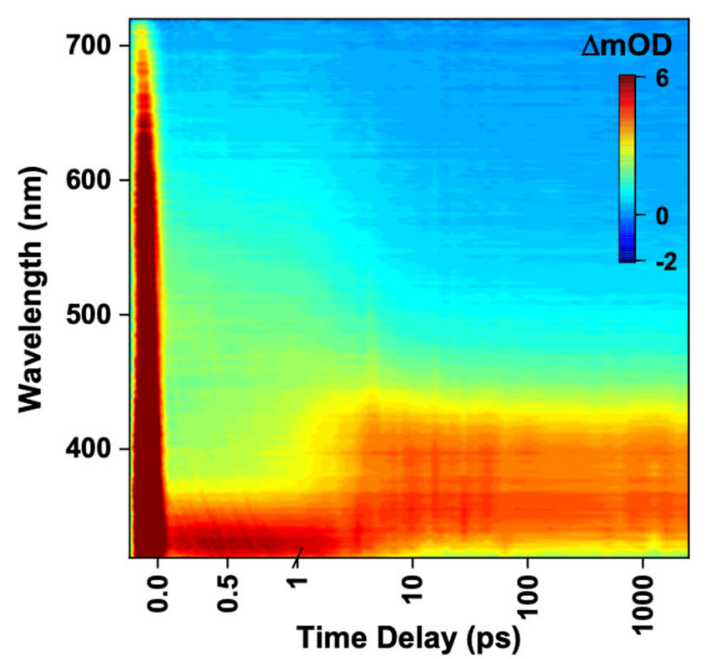

FIGURE 2 | TEA spectra represented as false color maps of $4 \mathrm{mM} \mathbf{A C y O}$ in (A) EtOH photoexcited at $285 \mathrm{~nm}$ and (B) DMSO photoexcited at $280 \mathrm{~nm}$. For (A,B) the time delays are plotted linearly until $1 \mathrm{ps}$ and then as a log scale from 1 to $2,500 \mathrm{ps}$.

the TEA spectra for ACyO in DMSO shown in Figure 2B. At early time delays, there is a broad ESA spanning from 320 to $720 \mathrm{~nm}$ peaking at $\sim 330 \mathrm{~nm}$. The broad ESA narrows and the greatest intensity red-shifts to center at $\sim 370 \mathrm{~nm}$ in around $5 \mathrm{ps}$. This feature then persists to the maximum $\Delta t$ of the experiment ( $\Delta t=2.5 \mathrm{~ns}$ ), see Supplementary Figure 6B for lineouts.

Although mentioned previously, it is worth revisiting the proposed cyclohexenone core deactivation pathway upon photoexcitation reported by Losantos et al. (2017) as it is the basis for the time constant assignment presented herein. The vertical excitation calculations that were carried out for the present study corroborate previous work which states that $\mathrm{ACyO}$ is initially photoexcited to the $S_{2}$ through a $\pi_{L}^{*} \leftarrow \pi_{H}$ transition (see Supplementary Table 4 and Supplementary Figure 7) (Sui et al., 2012; Losantos et al., 2017). The computed minimum energy path demonstrates that a similar molecule to $\mathrm{ACyO}\left(\mathrm{NHCH}_{3}\right.$ substituent on $\mathrm{C}_{3}$, herein termed the $\mathrm{NHCH}_{3}$ molecule, see inset of Figure 1) studied by Losantos et al. (2017) redistributes its energy after photoexcitation causing a geometric distortion. This leads to an accessible $S_{2} / S_{1}$ CI where it then vibrationally cools to a minimum on the $S_{1}$. Woolley et al. (2018) conducted complementary calculations on $\mathbf{A C y O}$ finding that the calculated energy barrier to the $\mathrm{S}_{1} / \mathrm{S}_{0} \mathrm{CI}$ for $\mathbf{A C y O}$ in the gas phase is $0.04 \mathrm{eV}$ (Woolley et al., 2018), cf. $0.2 \mathrm{eV}$ for the $\mathrm{NHCH}_{3}$ molecule studied by Losantos et al. (2017). We now assign the dynamical processes to the extracted time constants from the global sequential fit, re-evaluating our previous work in light of the present and previous data.

Global fitting of ACyO in EtOH extracted three time constants and can be found in Table 1; we add that as the negative feature is the result of multiphoton initiated dynamics, it has been omitted from the global fit. We propose that the first time constant, $\tau_{\mathrm{e} 1}=0.24 \mathrm{ps}$, corresponds to the initial geometry relaxation and solvent rearrangement $\mathbf{A C y O}$ undergoes in order to traverse the $S_{2} / S_{1} C I$ and populate the $S_{1}$ potential energy
TABLE 1 | Time constants and associated errors extracted from the sequential global fit of the TEA spectra for ACyO in EtOH and DMSO.

\begin{tabular}{lcc}
\hline Time constant & EtOH & DMSO \\
\hline$\tau_{\mathrm{e} 1}$ & $0.24 \pm 0.035 \mathrm{ps}$ & $2.7 \pm 0.038 \mathrm{ps}$ \\
$\tau_{\mathrm{e} 2}$ & $1.2 \pm 0.035 \mathrm{ps}$ & $>2.5 \mathrm{~ns}$ \\
$\tau_{\mathrm{e} 3}$ & $>2.5 \mathrm{~ns}$ & \\
\hline
\end{tabular}

surface. We attribute the second time constant, $\tau_{\mathrm{e} 2}=1.2 \mathrm{ps}$, with the vibrational cooling of $\mathbf{A C y O}$, via vibrational energy transfer (both intramolecular and intermolecular), as it samples the $S_{1}$ potential energy surface before overcoming the barrier to, and traversing through the $\mathrm{S}_{1} / \mathrm{S}_{0} \mathrm{CI}$. From the evolution associated difference spectra and lineouts of $\mathrm{ACyO}$ in $\mathrm{EtOH}$ (see Supplementary Figures 2A, 6A), narrowing of the ESA is observed which is a good indication that vibrational cooling on the $S_{1}$ potential energy surface is occurring (also the case for ACyO in DMSO, see Supplementary Figures 2B, 6B). We note here that as the ESA $(\sim 330 \mathrm{~nm})$ decays out of the probe window in our TEA spectra, it is possible that $\tau_{\mathrm{e} 2}$ is an underestimation of the time constant associated with repopulation of the electronic ground state. The final time constant, $\tau_{\mathrm{e} 3}>2.5 \mathrm{~ns}$, corresponds to any ACyO that has remained trapped in the minimum of the $\mathrm{S}_{1}$ excited state.

The global fitting of ACyO in DMSO extracted two time constants, see Table 1. We have assigned the first time constant, $\tau_{\mathrm{e} 1}=2.7 \mathrm{ps}$, to a collection of processes; geometry relaxation and solvent rearrangement ACyO undergoes to reach the $S_{2} / S_{1} C I$ (similar to $\tau_{\mathrm{e} 1}$ for $\mathbf{A C y O}$ in $\mathrm{EtOH}$ ), followed by vibrational energy transfer along the $S_{1}$ potential energy surface before repopulating the electronic ground state, mediated via the $\mathrm{S}_{1} / \mathrm{S}_{0} \mathrm{CI}$ (similar to $\tau_{\mathrm{e} 2}$ for $\mathbf{A C y O}$ in $\left.\mathrm{EtOH}\right)$. As with $\mathbf{A C y O}$ in $\mathrm{EtOH}$, the second time constant, $\tau_{\mathrm{e} 2}>2.5 \mathrm{~ns}$, corresponds to any $\mathrm{ACyO}$ that is still 
populated in the $S_{1}$ excited state beyond the final time delay of our experiment. Based on the above assignments, we attribute the ESA at $\sim 400 \mathrm{~nm}$ which grows in within $\sim 5$ ps and persists beyond the final time delay in the TEA spectra of both solvents (Figures 2A,B) to ESA from the $S_{1}$ minimum. For ACyO in DMSO, the ESA at extended time delays $(2.5 \mathrm{~ns})$ is still relatively large in intensity; however, from our TVA spectra we know that a large percentage of ACyO molecules have already returned to their electronic ground state within $\sim 5 \mathrm{ps}$, see later discussion. Plausible explanations are that the strength of the ESA from the $S_{1}$ minimum is greater than at other locations along the potential energy surface, or the ESA at early time delays is dampened by a competing stimulated emission. Thus, a reduced population on the $S_{1}$ minimum could (and evidently does) result in a more intense ESA. Further to this, we have determined through our TVA spectra that the relaxation mechanism in both solvents is the same, however, there is evidently a solvent dependence on the strength of the ESAs from different positions on the relaxation pathway. For example, the strength of the ESA from the $S_{1}$ minimum in EtOH is evidently weaker than the strength of the ESA from the $S_{1}$ minimum in DMSO. This highlights the influence of solvent on the oscillator strength, which was previously discussed by Woolley et al. (2018). Furthermore, our assignment here (and below) of population trapped on $S_{1}$ concurs with previous work in that there is a barrier to the $S_{1} / S_{0} C I$ (Losantos et al., 2017; Woolley et al., 2018).

However, we highlight again that no indication on the percentage of ACyO trapped in the $S_{1}$ excited state can be inferred from the TEA spectra. To glean further insight into this, we conducted both TVAS and steady-state irradiation studies. To begin the TVAS discussion, we must first look at the FTIR spectra of ACyO in EtOH and DMSO and assign the vibrational modes to the peaks of interest. Table 2 gives the averaged explicitsolvent calculated $\mathrm{S}_{0}$ frequencies (with associated scaling factors of 0.997 and 0.982 for EtOH and DMSO, respectively) and associated vibrational modes. Figure 3 is a visual representation of the calculated frequencies overlaying the FTIR spectra for each ACyO solution between 1,500 and $1,750 \mathrm{~cm}^{-1}$. Only this region of the FTIR spectra is displayed as it is the region that is not masked by solvent absorption and includes the strongest peaks. The relative strengths of the vibrations have also been scaled to match the reference peaks at 1,559 and $1,577 \mathrm{~cm}^{-1}$ for ACyO in EtOH and DMSO, respectively.

The FTIR spectra for ACyO in EtOH and DMSO have many differences such as: peak positions; peak widths; peak intensities; and, to a lesser extent, assigned vibrational modes. This highlights how solvent environment effects the observed FTIR spectra and provides a further justification for our choice to implement explicit-solvent calculations. Similarities between the two spectra are that the strongest peak in each spectrum corresponds to the $\mathrm{C}_{2}=\mathrm{C}_{3}$ stretch (given in Table 2 are the additional vibrational modes for this peak in each solvent but for simplicity it is referred to as the $\mathrm{C}_{2}=\mathrm{C}_{3}$ stretch herein) and the weaker peaks at highest wavenumbers in the presented spectra in Figure 3 correspond to the $\mathrm{H}_{16}-\mathrm{N}_{8}-\mathrm{H}_{17}$ scissor mode, see inset of Figure $\mathbf{1}$ for atom numbers. A similar pattern was observed in a previous study which computed the vibrational frequencies for ACyO in water and acetonitrile adding further credence to our assignment (Sui et al., 2012).

Generally, the calculated peak positions are in good agreement with the experimental data with the largest error being $<20 \mathrm{~cm}^{-1}$ for the majority of the calculated frequencies. The only exception to this is the calculated frequency of $1,647 \mathrm{~cm}^{-1}$ for ACyO in DMSO which is shifted $\sim 45 \mathrm{~cm}^{-1}$ to a higher wavenumber compared to the experimental peak. This could be explained by the fact we only consider two DMSO molecules interacting with ACyO in our calculations compared to a full solvation shell interaction between solute and solvent. However, due to the reasonable accuracy for all other frequencies and because this vibrational mode does not have a corresponding $S_{1}$ frequency (see later discussion), the computational expenditure required for a full solvent shell calculation is deemed unnecessary.

The TVA spectra for ACyO in EtOH and DMSO and their associated tri-exponential fits for the GSB are shown in Figure 4. Global fitting was not implemented here as we were only interested in the GSB recovery of the probed vibrational modes. Furthermore, we note that our tri-exponential fits begin at the GSB signal maximum; for ACyO in $\mathrm{EtOH}$ this was 0.4 ps, and

TABLE 2 | Computed $S_{0}$ and $S_{1}$ vibrational frequencies and their associated vibrational modes at the PBEO/cc-pVTZ level of theory for ACyO in EtOH and DMSO between 1,500 and $1,750 \mathrm{~cm}^{-1}$.

\begin{tabular}{|c|c|c|c|c|}
\hline Solvent & $\begin{array}{l}\mathrm{S}_{0} \text { frequencies, } \mathrm{cm}^{-1} \\
\text { (Rel. strength) }\end{array}$ & $\begin{array}{l}\text { Vibrational mode } \\
\mathrm{S}_{0}\end{array}$ & $\begin{array}{l}\mathrm{S}_{1} \text { frequencies, } \mathrm{cm}^{-1} \\
\text { (Rel. strength) }\end{array}$ & $\begin{array}{l}\text { Vibrational mode } \\
\mathrm{S}_{1}\end{array}$ \\
\hline \multirow[t]{3}{*}{$\mathrm{EtOH}$} & $1,559(298)$ & $\begin{array}{l}\mathrm{C}_{2}=\mathrm{C}_{3} \text { stretch }+\mathrm{C}_{1}=\mathrm{O}_{7} \text { stretch }+ \\
\mathrm{C}_{2}-\mathrm{H}_{9} \text { bend }\end{array}$ & $1,517(12)$ & $\mathrm{H}_{10}-\mathrm{C}_{4}-\mathrm{H}_{11}$ scissor \\
\hline & $1,605(4)$ & $\begin{array}{l}\mathrm{C}_{2}=\mathrm{C}_{3} \text { stretch }+\mathrm{C}_{1}=\mathrm{O}_{7} \text { stretch }+ \\
\mathrm{H}_{16}-\mathrm{N}_{8}-\mathrm{H}_{17} \text { scissor }\end{array}$ & $1,565(46)$ & $\begin{array}{l}\mathrm{C}_{2}=\mathrm{C}_{3} \text { stretch }+\mathrm{H}_{10}-\mathrm{C}_{4}-\mathrm{H}_{11} \text { scissor } \\
+\mathrm{N}_{8}-\mathrm{H}_{17} \text { bend }+\mathrm{C}_{2}-\mathrm{H}_{9} \text { bend }\end{array}$ \\
\hline & $1,685(27)$ & $\mathrm{H}_{16}-\mathrm{N}_{8}-\mathrm{H}_{17}$ scissor & $1,714(34)$ & $\mathrm{H}_{16}-\mathrm{N}_{8}-\mathrm{H}_{17}$ scissor \\
\hline \multirow[t]{3}{*}{ DMSO } & $1,577(185)$ & $\begin{array}{l}\mathrm{C}_{2}=\mathrm{C}_{3} \text { stretch }+\mathrm{C}_{4}-\mathrm{H}_{10} \text { bend }+ \\
\mathrm{N}_{8}-\mathrm{H}_{17} \text { bend }+\mathrm{C}_{2}-\mathrm{H}_{9} \text { bend }\end{array}$ & $1,523(91)$ & $\begin{array}{l}\mathrm{C}_{2}=\mathrm{C}_{3} \text { stretch }+\mathrm{C}_{4}-\mathrm{H}_{10} \text { bend }+ \\
\mathrm{N}_{8}-\mathrm{H}_{17} \text { bend }+\mathrm{C}_{2}-\mathrm{H}_{9} \text { bend }\end{array}$ \\
\hline & $1,647(111)$ & $\mathrm{C}_{1}=\mathrm{O}_{7}$ stretch $+\mathrm{H}_{16}-\mathrm{N}_{8}-\mathrm{H}_{17}$ scissor & No corresponding frequency & \\
\hline & $1,675(9)$ & $\mathrm{H}_{16}-\mathrm{N}_{8}-\mathrm{H}_{17}$ scissor & $1,645(44)$ & $\mathrm{H}_{16}-\mathrm{N}_{8}-\mathrm{H}_{17}$ scissor \\
\hline
\end{tabular}

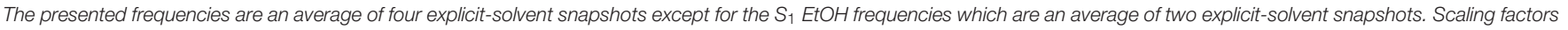
of 0.997 and 0.982 were applied to the calculated frequencies for $\mathbf{A C y O}$ in EtOH and DMSO, respectively. See text for details. 

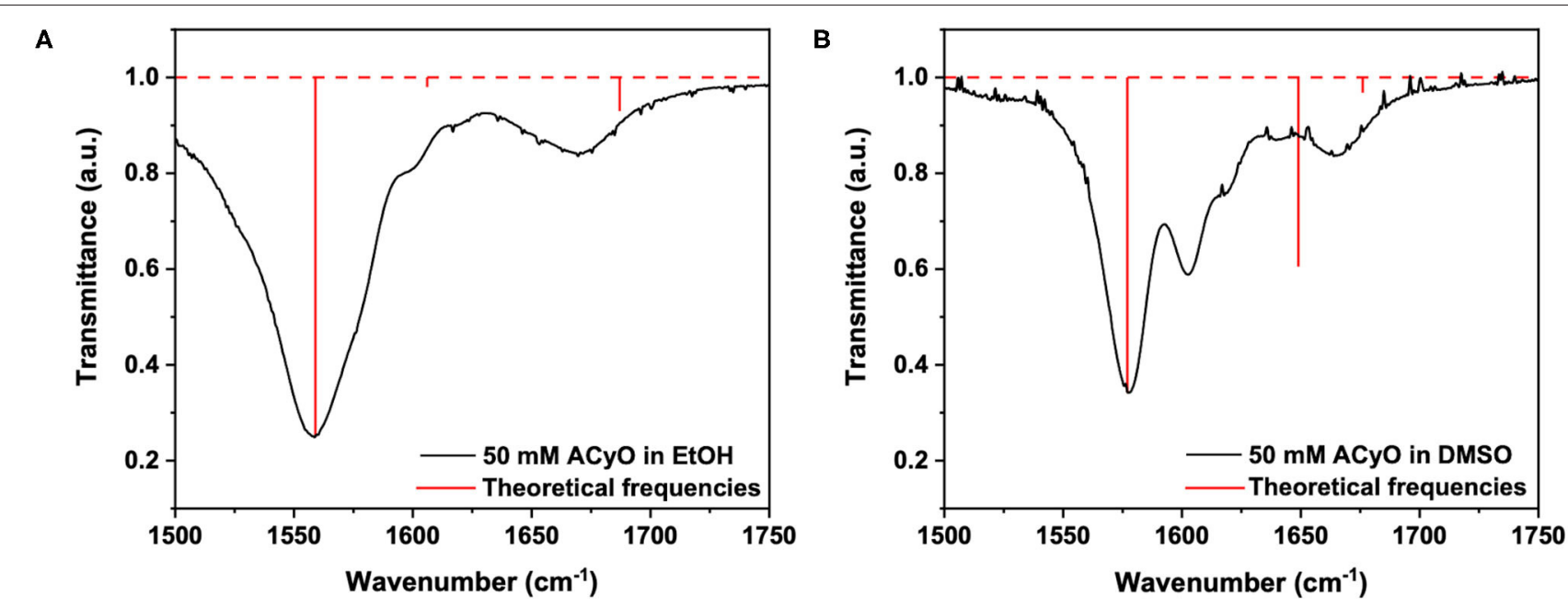

FIGURE 3 | Steady-state FTIR spectra (black lines) of 50 mM ACyO in (A) EtOH and (B) DMSO over 1,500-1,750 cm ${ }^{-1}$. Overlaying the FTIR spectra are the predicted frequencies of 4 averaged explicit-solvent snapshots for each solvent computed at the PBEO/cc-pVTZ level of theory and scaled with the scaling factors of 0.997 and 0.982, respectively, for ACyO in EtOH and DMSO (see text for details). The predicted vibrational frequencies are represented by red vertical lines.

for ACyO in DMSO this was 0.7 ps. In fitting this way, we avoid any coherent artifacts that occur at early time delays such as perturbed free induction decay (Hamm, 1995). We start the TVAS discussion by first highlighting that in both solvents, full GSB recovery is not achieved at $\Delta t=1.8 \mathrm{~ns}$. This is in accordance with the TEA spectra supra which show excited state dynamics persisting beyond $\Delta t=2.5 \mathrm{~ns}$. The extracted GSB recovery quantum yields from the integrated signal of each GSB feature were calculated to be 86 and $76 \%$ for ACyO in $\mathrm{EtOH}$ (over $1,540-1,550 \mathrm{~cm}^{-1}$, see later discussion for our choice to use this integration region) and DMSO (over 1,560-1,585 $\mathrm{cm}^{-1}$ ) respectively. Greater GSB recovery is observed for ACyO in EtOH compared to ACyO in DMSO by $10 \%$. A similar trend was observed for the long-term irradiation studies of ACyO presented below. The two are intuitively related and imply that more ACyO molecules in $\mathrm{EtOH}$ are able to overcome the barrier to access the $\mathrm{S}_{1} / \mathrm{S}_{0} \mathrm{CI}$ within $\Delta t=1.8 \mathrm{~ns}$. Therefore, there is less population trapped in the electronic excited state that can undergo pathways leading to photodegradation compared to ACyO in DMSO. We note here that the GSB recovery quantum yields we quote only consider GSB recovery that occurs within $1.8 \mathrm{~ns}$, and it is possible that further electronic ground state repopulation may occur after that time.

The time constants extracted from the fit for ACyO in $\mathrm{EtOH}$ $\left(1,559 \mathrm{~cm}^{-1}\right.$ peak) and DMSO $\left(1,577 \mathrm{~cm}^{-1}\right.$ peak $)$ are reported in Table 3. We begin by assigning the dynamical processes of the extracted time constants for ACyO in EtOH. The first time constant, $\tau_{\mathrm{v} 1}=0.53 \mathrm{ps}$, is on a similar timescale to $\tau_{\mathrm{e} 2}$ from the TEA spectra fit which would suggest that similar dynamics are occurring as we have reported; geometry relaxation and solvent rearrangement of $\mathbf{A C y O}$, followed by vibrational energy transfer on $S_{1}$ and then repopulation of $S_{0}$. However, as TVAS only measures the GSB recovery of the fundamental vibration (being probed) in the electronic ground state, it provides little information about the dynamics in the electronic excited state, making comparison between $\tau_{\mathrm{e} 2}$ and $\tau_{\mathrm{v} 1}$ tentative. As a result, we note that $\tau_{\mathrm{v} 1}$ may only be incorporating some of the electronic excited state dynamics and repopulation of the electronic ground state is most likely occurring on a longer time scale. The second time constant for the TVA spectra, $\tau_{\mathrm{v} 2}=49 \mathrm{ps}$, is associated with the vibrational cooling of the electronic ground state through vibrational energy transfer, both intramolecular and intermolecular (to solvent). This longer timescale can only be observed in the TVA spectra as the GSB of ACyO falls outside of the probe window for our TEAS experiment. As a result, no spectral signatures of vibrational cooling on the $S_{0}$ are present in the TEA spectra, and thus no time constant can be assigned to this photophysical process. Furthermore, we do not observe a blue-shifting of the transient vibrational bands with time that is often associated with the anharmonicity of vibrations (Nibbering et al., 2005), and instead observe a slight red-shift for $\mathrm{ACyO}$ in $\mathrm{EtOH}$ (Figure 4A). We reconcile this through the following: firstly, it is possible that the probed vibration is relatively harmonic; and secondly, the ESA from the $\mathrm{S}_{1}$ minimum between $\sim 1,560$ and $1,570 \mathrm{~cm}^{-1}$, which contributes toward the GSB recovery and causes an asymmetric line shape at extended time delays (see later discussion), is likely the main cause of the apparent red-shift. For ACyO in DMSO (Figure 4B), neither a red-shift or a blue-shift is observed, providing confidence that the probed vibrations are relatively harmonic. The final time constant for $\mathrm{ACyO}$ in $\mathrm{EtOH}, \tau_{\mathrm{v} 3}>1.8 \mathrm{~ns}$, is due to the incomplete GSB recovery. Drawing reference to our TEAS data, this is very likely attributed to the long-lived ACyO trapped in the minimum of the $S_{1}$.

The calculated $S_{1}$ frequencies and associated vibrational modes for ACyO in EtOH can be found in Table 2 and were scaled using the same scaling factor that was applied to the $\mathrm{S}_{0}$ frequencies, 0.997. The reported frequencies are an average 

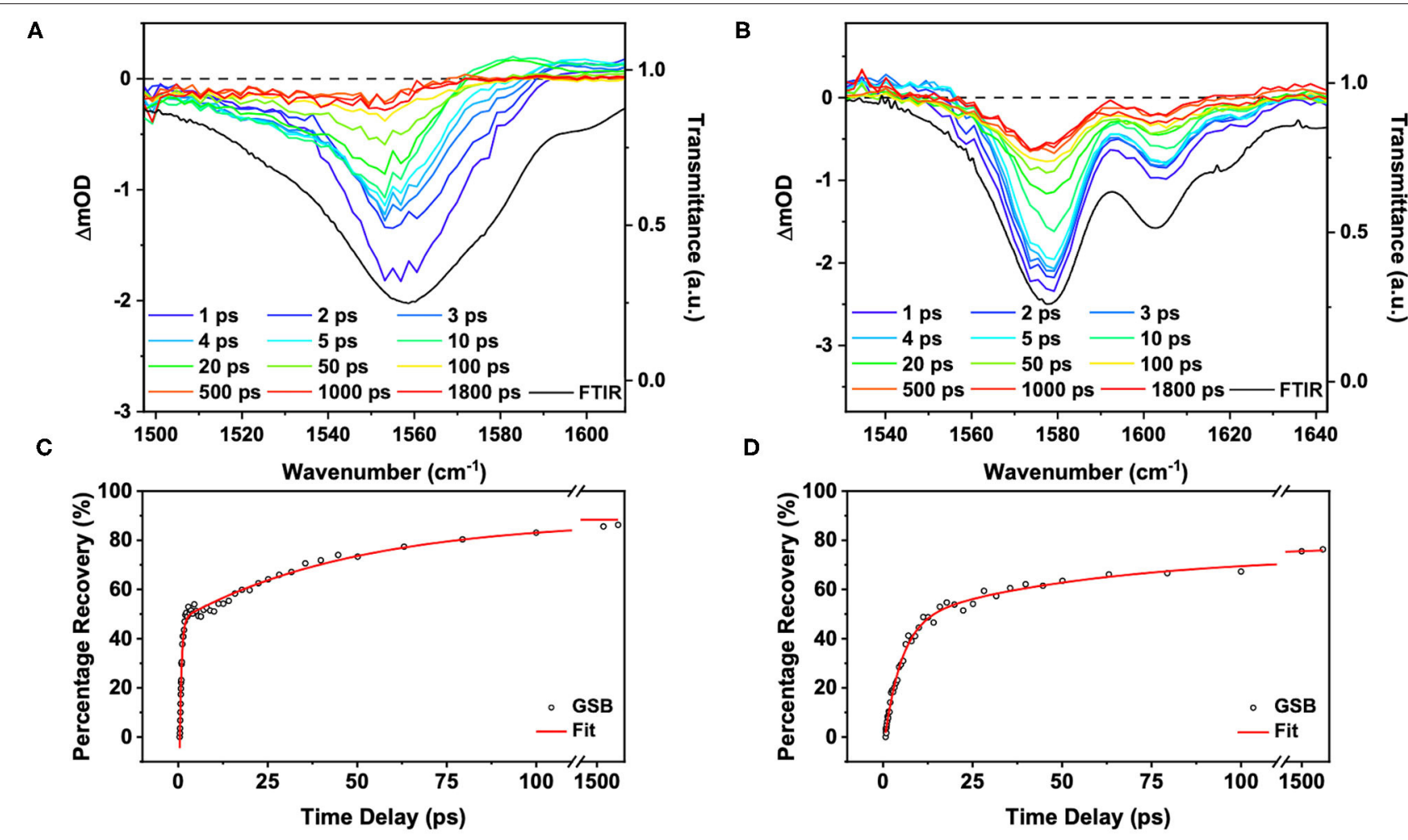

FIGURE 4 | TVA spectra at selected time delays and steady-state FTIR of $50 \mathrm{mM}$ ACyO in (A) EtOH photoexcited at $285 \mathrm{~nm}$ and (B) DMSO photoexcited at $280 \mathrm{~nm}$. Scale on left of plots correspond to the change in optical density for the TVA spectra (colored lines) and scale of the right corresponds to the transmittance for the FTIR spectra (black lines). (C) Tri-exponential fit of the percentage recovery of the integrated GSB signal from the TVA spectra for ACyO in EtOH (1,540-1,550 $\mathrm{cm}^{-1}$ ). (D) Tri-exponential fit of the percentage recovery of the integrated GSB signal from the TVA spectra for $\mathbf{A C y O}$ in DMSO (1,560-1,585 $\mathrm{cm}^{-1}$ ). In both (C,D), the open circles are the integrated GSB signal converted to percentage recovery and the red line is the fit. The time delays are plotted linearly until 110 ps then there is a break until 1,300 ps. Between 1,300 and 1,900 ps the scale is plotted logarithmically to show that the fit does not tend to zero.

TABLE 3 | Time constants and associated errors extracted from the tri-exponential fit of the integrated GSB signal from the TVA spectra for ACyO in EtOH (1,540-1,550 $\left.\mathrm{cm}^{-1}\right)$ and DMSO (1,560-1,585 $\left.\mathrm{cm}^{-1}\right)$.

\begin{tabular}{lcc}
\hline Time constant & EtOH $\left(\mathbf{1}, \mathbf{5 5 9} \mathbf{~ c m}^{-\mathbf{1}}\right)$ & DMSO $\left(\mathbf{1}, \mathbf{5 7 7} \mathbf{~ m}^{\mathbf{- 1}}\right)$ \\
\hline$\tau_{\mathrm{v} 1}$ & $0.53 \pm 0.028 \mathrm{ps}$ & $4.7 \pm 0.35 \mathrm{ps}$ \\
$\tau_{\mathrm{v} 2}$ & $49 \pm 2.9 \mathrm{ps}$ & $55 \pm 9.0 \mathrm{ps}$ \\
$\tau_{\mathrm{v} 3}$ & $>1.8 \mathrm{~ns}$ & $>1.8 \mathrm{~ns}$ \\
\hline
\end{tabular}

of two snapshots due to difficulties experienced when running our EtOH explicit-solvent excited state calculations, see the Supplementary Material for more details. We draw confidence on the validity of these results from the similarity between the two snapshots, alongside the consistency of the results observed in DMSO. Future studies may invest further into converging more snapshots, but this has a high computational cost and is beyond the scope of the work. The only $S_{1}$ frequency which had a clear corresponding $S_{0}$ frequency between 1,500 and $1,750 \mathrm{~cm}^{-1}$ for ACyO in EtOH was for the $\mathrm{H}_{16}-\mathrm{N}_{8}-\mathrm{H}_{17}$ scissor vibrational mode. This mode is shifted $\sim 30 \mathrm{~cm}^{-1}$ in the $S_{1}$ to a higher wavenumber of $1,714 \mathrm{~cm}^{-1}$. Additionally, two other excited state frequencies were found during the calculation in the region of interest. These were computed as 1,517 and $1,565 \mathrm{~cm}^{-1}$ and they correspond to the $\mathrm{H}_{10}-\mathrm{C}_{4}-\mathrm{H}_{11}$ scissor and the $\mathrm{C}_{2}=\mathrm{C}_{3}$ stretch (plus several other vibrational modes), respectively, see Table 2 for full vibrational mode assignment. These vibrational modes are convoluted on top of the GSB of the $\mathrm{C}_{2}=\mathrm{C}_{3}$ stretch at 1559 $\mathrm{cm}^{-1}$ that we probed in the TVAS experiment. The relative strengths of the two $S_{1}$ frequencies are significantly smaller than the $S_{0}$ frequency therefore, we would expect any ESAs to be much weaker in intensity compared to the GSB. That being said, from the TVA spectra in Figure 4, a small ESA feature is observable at a wavenumber of $\sim 1,570 \mathrm{~cm}^{-1}$ and above; present between $10-20$ ps and decaying by $50 \mathrm{ps}$. We are unable to definitely pinpoint the origin of this feature. Possible suggestions include probing: (1) the $\mathrm{C}_{2}=\mathrm{C}_{3}$ stretch in $\mathrm{S}_{1}$; and (2) a higher lying vibration in ACyO in the ground electronic state which subsequently cools. Indeed, the decay of this feature matches $\tau_{\mathrm{v} 2}$ adding credence to the latter assignment. Importantly, at $1.8 \mathrm{~ns}$, the asymmetry of the GSB feature insinuates that an ESA is contributing toward the GSB recovery between $\sim 1,560$ and $1,570 \mathrm{~cm}^{-1}$. This matches the computed $S_{1}$ frequency at $1,565 \mathrm{~cm}^{-1}$, therefore, we assign it to the population of ACyO trapped in the $S_{1}$ minimum. The ESA does not emerge above baseline due to the fact the transition is six 
times weaker than the probed electronic ground state transition, see Table 2. Due to the convolution of peaks, we highlight that for the GSB recovery quantum yield and fitting of the GSB, a smaller region $\left(1,540-1,550 \mathrm{~cm}^{-1}\right)$ was integrated between to negate as many contributions from the overlapping ESAs as possible. We appreciate that there is still a possibility that the ESA is contributing to the GSB recovery, hence we report the GSB recovery quantum yield of $86 \%$ as being the upper limit in this case.

The TVA spectra of ACyO in DMSO is more straightforward to interpret due to no convoluted $S_{1}$ frequencies computed by TDDFT calculations. A tri-exponential fit of the integrated signal of the $1,577 \mathrm{~cm}^{-1}$ vibration $\left(1,560-1,585 \mathrm{~cm}^{-1}\right)$ can be found in Figure 4 and its corresponding time constants can be found in Table 3. The same assignment of the time constants is made for ACyO in DMSO as that reported above for ACyO in EtOH. $\tau_{\mathrm{v} 1}(4.7 \mathrm{ps})$ is assigned to the geometry relaxation and solvent rearrangement ACyO undergoes to access the $S_{2} / S_{1} C I$, followed by vibrational cooling on the $S_{1}$ and traversing through the $S_{1} / S_{0}$ CI to repopulate the electronic ground state. Like in ACyO in EtOH, the timescales of $\tau_{\mathrm{e} 1}$ and $\tau_{\mathrm{v} 1}$ are similar. $\tau_{\mathrm{v} 2}$ (55 ps) is assigned to the vibrational cooling of ACyO on the $\mathrm{S}_{0}$ through vibrational energy transfer. Again, comparison to the TEA spectra cannot be made for $\tau_{\mathrm{v} 2}$ due to TEAS experimental limitations as discussed above. The final time constant, $\tau_{\mathrm{v} 3}>1.8$ $\mathrm{ns}$, is assigned to any $\mathbf{A C y O}$ molecules trapped in the minimum of the $S_{1}$ potential energy surface.

We conclude the TVAS discussion by evaluating the $S_{1}$ vibrational frequency calculations for ACyO in DMSO which can be found in Table 2. These computed frequencies are an average of four explicit-solvent snapshots and were simpler to assign to their corresponding electronic ground state frequencies, unlike ACyO in EtOH. For the $\mathrm{C}_{2}=\mathrm{C}_{3}$ stretch vibration, calculated frequency values of $1,577 \mathrm{~cm}^{-1}$ in the $S_{0}$ state and 1,523 $\mathrm{cm}^{-1}$ in the $\mathrm{S}_{1}$ state are reported in Table 2. For the $\mathrm{H}_{16}$ $\mathrm{N}_{8}-\mathrm{H}_{17}$ scissor vibration, the calculated frequency values are $1,675 \mathrm{~cm}^{-1}$ in the $S_{0}$ state and $1,645 \mathrm{~cm}^{-1}$ in the $S_{1}$ state. Therefore, upon $S_{1} \leftarrow S_{0}$ excitation, the harmonic frequencies of both vibrations are shifted to a lower wavenumber; the frequency of the $\mathrm{C}_{2}=\mathrm{C}_{3}$ stretch vibration by $\sim 50 \mathrm{~cm}^{-1}$ and the frequency of the $\mathrm{H}_{16}-\mathrm{N}_{8}-\mathrm{H}_{17}$ scissor vibration by $\sim 30 \mathrm{~cm}^{-1}$. There was no corresponding $\mathrm{S}_{1}$ frequency for the $\mathrm{C}_{1}=\mathrm{O}_{7}$ stretch plus $\mathrm{H}_{16}-\mathrm{N}_{8}-\mathrm{H}_{17}$ scissor that was computed as $1,647 \mathrm{~cm}^{-1}$ in the electronic ground state. Additionally, there were no other new $S_{1}$ frequencies appearing between 1,500 and $1,750 \mathrm{~cm}^{-1}$ and this insinuates that no ESA features should be present in the TVA spectra, which is the case as seen in Figure 4B. As a result, we are confident that the GSB recovery quantum yield for ACyO in DMSO (76\%) is a more accurate value than the GSB recovery quantum yield for ACyO in $\mathrm{EtOH}$ (86\%). We add that a degenerate pump-probe experiment would complement the observed GSB recovery quantum yields obtained from our TVAS experiments; however, for the present study, the TVAS data provides a good starting point with which to progress with future studies.

In our efforts to link our ultrafast dynamics studies of ACyO (and the GSB recovery quantum yields thereof) to the long-term photostability of ACyO, we performed long-term irradiation studies and the results are presented in Figure 5. The data shows that after $7,200 \mathrm{~s}$, only 2.0 and $6.5 \%$ degradation of signal at the absorption maxima occurred for ACyO in $\mathrm{EtOH}$ and DMSO, respectively. Control measurements confirmed that all degradation observed was due to photodegradation, see Supplementary Figure 8. The impressive levels of photostability of ACyO in both solvents must stem from highly efficient repopulation of the electronic ground state as we observed in our TVAS studies supra. In the long-term irradiation study, we see no emergence of an absorbance at $\sim 400 \mathrm{~nm}$ in either solvent, eliminating the possibility that the long-lived feature in the TEA spectra is a photoproduct. This provides further support that the long-lived feature observed in our TEA and TVA spectra is ACyO population trapped in $S_{1}$ state. Losantos et al. (2017) predicted that the cyclohexenone unit they studied would radiatively decay after becoming trapped on the $S_{1}$ in order to repopulate the molecules' electronic ground state. However, in accordance with Woolley et al. (2018) we observed no radiative decay implying that ACyO must recover to the electronic ground state non-radiatively. We draw this conclusion from the fact that no stimulated emission features (induced by one-photon absorption of the pump) are present in the TEA spectra for ACyO in both EtOH and DMSO, and that no emission was observed in the steady-state emission spectra of ACyO in both solvents when compared to blank scans of solvent-only solutions (spectra not shown). The lack of observed fluorescence would suggest that there is very poor Franck-Condon overlap between the $S_{1}$ minimum and the electronic ground state. These steadystate results further support our conclusion that the $S_{1} / S_{0} C I$ is accessed by $>75 \%$ of ACyO molecules on ultrafast timescales as they are able to overcome the mild barrier from the $\mathrm{S}_{1}$ minimum (0.04 eV) (Woolley et al., 2018).

For the ACyO population remaining in the excited state at extended time delays, $>1.8 \mathrm{~ns}$, it is likely that further repopulation of the electronic ground state and some photoproduct formation occurs. It is important to highlight that the systems we have studied are simple, isolated systems, therefore, we have observed repopulation of the electronic ground state at time beyond $1.8 \mathrm{~ns}$. However, in a more complex environment like a sunscreen formulation, the population of ACyO in the $S_{1}$ excited state persisting beyond $1.8 \mathrm{~ns}$ has a greater chance of either forming a photoproduct or transferring that energy through collisions. This reinforces the importance of why an ideal UV filter would return to its electronic ground state on ultrafast timescales and why we conclude that ACyO is not a perfect UV filter candidate. We close the discussion by reiterating that more photoproduct formation appears to be occurring for ACyO in DMSO which is intuitively related to the lower GSB recovery quantum yield extracted from the TVA spectra presented above. The greater population persisting in the $S_{1}$ excited state at extended time delays, $>1.8 \mathrm{~ns}$, increases the probability for photoproduct formation.

\section{CONCLUSION}

Throughout the present work we have demonstrated that although ACyO has a long-lived excited state that persists 
A

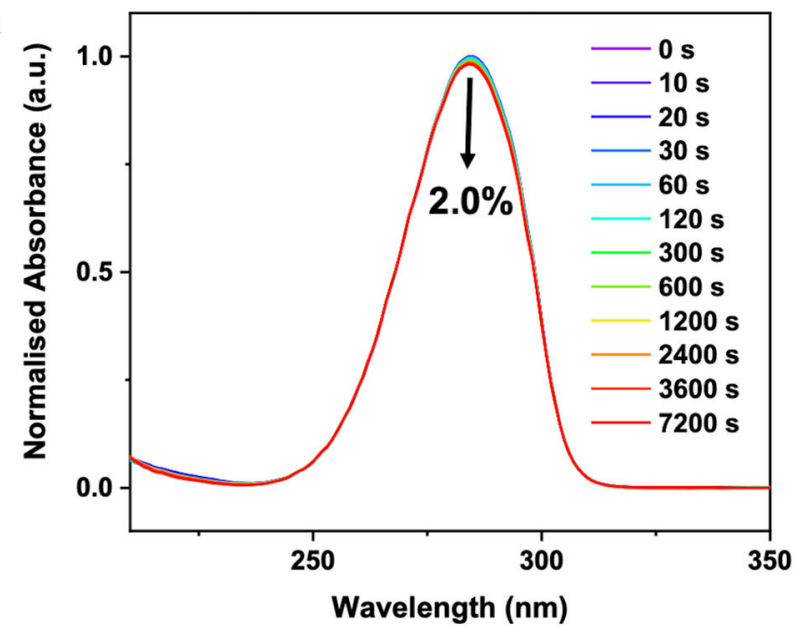

B

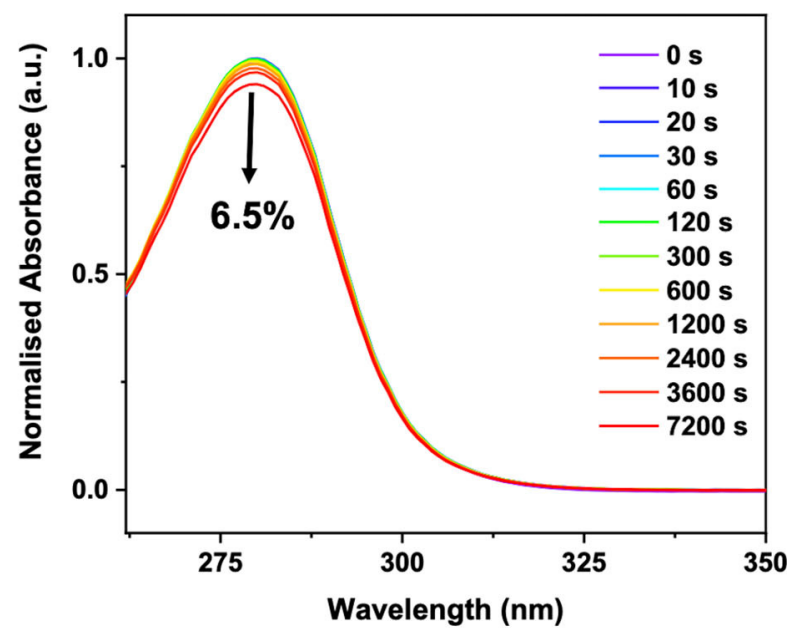

FIGURE 5 | UV/visible spectra of $\sim 40 \mu \mathrm{M}$ ACyO in (A) EtOH and (B) DMSO taken over varying durations of irradiation in a solar simulator. Black arrow on each plot denotes a decrease in absorbance at the absorption maxima after 7,200 s and corresponds to $2.0 \%$ for $\mathbf{A C y O}$ in EtOH and 6.5\% for ACyO in DMSO.

beyond $2.5 \mathrm{~ns}$, the extent of ground state recovery on ultrafast timescales is relatively high. We have determined that $>75 \%$ of photoexcited ACyO in EtOH and DMSO is returning to its electronic ground state within 1.8 ns. These numbers appear to corroborate why ACyO displays good photostability in the isolated systems studied. Upon photoexcitation, we demonstrate that the majority of $\mathbf{A C y O}$ molecules have sufficient energy to overcome the $S_{1} / S_{0} C I$ barrier and relax nonradiatively on ultrafast timescales. Although $\mathrm{ACyO}$ displays longlived features in accordance with previous studies (Losantos et al., 2017; Woolley et al., 2018), we propose that the cyclohexenone core is not as poor a UV filter as initially predicted, supporting nature's choice of the cyclohexenone core in MAAs. However, for an ideal UV filter we would ideally like as-close-to complete GSB recovery within ultrafast timescales; further studies on molecules with a cyclohexenone unit are therefore most-certainly warranted so as to provide clearer insight in to their photoprotective mechanisms, and how these mechanisms are influenced with substituent. In particular, how the GSB recovery quantum yield is influenced as we move progressively toward gadusol is one avenue to be explored, which could eventually guide the future design of UV filters based on MAAs.

In this present work, we have demonstrated that TVAS is a very powerful method when allied with appropriate highlevel theory (in this case using explicit-solvent methodology which is important to consider when solute and solvent interact strongly with each other) for extracting accurate GSB recovery information. The combination of this high-level theory and TVAS enables vibrational ESA bands to be identified when selecting an integration region, allowing for the origins of a molecule's photostability to be traced. TVAS provides invaluable dynamical information that cannot be extracted from TEAS. Therefore, we suspect that this technique will see increasing use in the field of sunscreen science.

\section{DATA AVAILABILITY STATEMENT}

The datasets presented in this study can be found in online repositories. The names of the repository/repositories and accession number(s) can be found below: Zenodo repository doi: 10.5281/zenodo.3885450.

\section{AUTHOR CONTRIBUTIONS}

ALW conducted the majority of the experimental and computational work and took lead in writing the manuscript. MAPT ran the molecular dynamics simulations and provided support for the computational analysis. MAPT and JMW supervised the writing of the manuscript. DJLC and MDH helped conduct the transient vibrational absorption spectroscopy experiments presented in this paper. VGS conceived the experiment, supervised the writing of the manuscript, and supported the data analysis. All authors contributed to the article and approved the submitted version.

\section{ACKNOWLEDGMENTS}

The authors would like to thank the Warwick Centre for Ultrafast Spectroscopy (WCUS) for the use of the VERTEX 70v and ultrafast spectroscopy equipment. ALW thanks the University of Warwick and Lubrizol for funding a Ph.D. studentship through the Centre for Doctoral Training in Analytical Science. MAPT thanks the EPSRC for a doctoral studentship through the EPSRC Centre for Doctoral Training in Molecular Analytical Science, Grant No. EP/L015307/1. DJLC would like to acknowledge financial support from EPSRC under grant EP/N010825/1, and EPSRC and the De Beers Group facilitated through the Center for Doctoral Training in Diamond Science and Technology. JMW is grateful to EPSRC and Newport Spectra-Physics Ltd for a joint studentship. MDH thanks the Leverhulme Trust for 
postdoctoral funding. VGS thanks the EPSRC for an equipment grant (EP/J007153), the Leverhulme Trust for a research grant (RPG-2016-055) and the Royal Society and Leverhulme Trust for a Royal Society Leverhulme Trust Senior Research Fellowship. This project has received funding from the European Union's Horizon 2020 research and innovation programme under the grant agreement No. 828753. Computing facilities were provided by the Scientific Computing Research Technology Platform of the University of Warwick. We acknowledge the use of

\section{REFERENCES}

Adamo, C., and Barone, V. (1999). Toward reliable density functional methods without adjustable parameters: the PBE0 model. J. Chem. Phys. 110:6158. doi: 10.1063/1.478522

Arbeloa, E. M., Bertolotti, S. G., and Churio, M. S. (2011). Photophysics and reductive quenching reactivity of gadusol in solution. Photochem. Photobiol. Sci. 10, 133-142. doi: 10.1039/C0PP00250J

Baker, L. A., Horbury, M. D., Greenough, S. E., Allais, F., Walsh, P. S., Habershon, S., et al. (2016). Ultrafast photoprotecting sunscreens in natural plants. J. Phys. Chem. Lett. 7, 56-61. doi: 10.1021/acs.jpclett.5b02474

Baker, L. A., Horbury, M. D., Greenough, S. E., Coulter, P. M., Karsili, T. N. V., Roberts, G. M., et al. (2015). Probing the ultrafast energy dissipation mechanism of the sunscreen oxybenzone after UVA irradiation. J. Phys. Chem. Lett. 6, 1363-1368. doi: 10.1021/acs.jpclett.5b00417

Baker, L. A., Staniforth, M., Flourat, A. L., Allais, F., and Stavros, V. G. (2018). Gas-solution phase transient absorption study of the plant sunscreen derivative methyl sinapate. ChemPhotoChem 2, 743-748. doi: 10.1002/cptc.201800060

Balskus, E. P., and Walsh, C. T. (2010). The genetic and molecular basis for sunscreen biosynthesis in cyanobacteria. Science 329, 1653-1656. doi: 10.1126/science.1193637

Bandaranayake, W. M. (1998). Mycosporines: are they nature's sunscreens? Nat. Prod. Rep. 15, 159-172. doi: 10.1039/a815159y

Brenner, M., and Hearing, V. J. (2008). The protective role of melanin against UV damage in human skin. Photochem. Photobiol. 84, 539-549. doi: 10.1111/j.1751-1097.2007.00226.x

Bryden, A. M., Moseley, H., Ibbotson, S. H., Chowdhury, M. M., Beck, M. H., Bourke, J., et al. (2006). Photopatch testing of 1155 patients: results of the U.K. multicentre photopatch study group. Br. J. Dermatol. 155, 737-747. doi: 10.1111/j.1365-2133.2006.07458.x

Case, D. A., Berryman, J. T., Betz, R. M., Cerutti, D. S., Cheatham, T. E. III, Darden, T.A., et al. (2015). AMBER 2015. University of California: San Francisco, CA.

Casida, M. E. (1995). "Time-dependent density functional response theory for molecules," in Recent Advances in Density Functional Methods, ed D. P. Chong (Singapore: World Scientific), 155-192.

Conde, F. R., Churio, M. S., and Previtali, C. M. (2000). The photoprotector mechanism of mycosporine-like amino acids. Excited-state properties and photostability of porphyra-334 in aqueous solution. J. Photochem. Photobiol. B Biol. 56, 139-144. doi: 10.1016/S1011-1344(00)00066-X

Conde, F. R., Churio, M. S., and Previtali, C. M. (2007). Experimental study of the excited-state properties and photostability of the mycosporine-like amino acid palythine in aqueous solution. Photochem. Photobiol. Sci. 6, 669-674. doi: 10.1039/b618314j

Danovaro, R., Bongiorni, L., Corinaldesi, C., Giovannelli, D., Damiani, E., Astolfi, P., et al. (2008). Sunscreens cause coral bleaching by promoting viral infections. Environ. Health Perspect. 116, 441-447. doi: 10.1289/ehp.10966

Davidson, E. R. (1996). Comment on "comment on Dunning's correlation-consistent basis sets". Chem. Phys. Lett. 260, 514-518. doi: 10.1016/0009-2614(96)00917-7

Dean, J. C., Kusaka, R., Walsh, P. S., Allais, F., and Zwier, T. S. (2014). Plant sunscreens in the UV-B: ultraviolet spectroscopy of jet-cooled sinapoyl malate, sinapic acid, and sinapate ester derivatives. J. Am. Chem. Soc. 136, 14780-14795. doi: $10.1021 /$ ja5059026
Athena at HPC Midlands+, which was funded by the EPSRC by Grant No. EP/P020232/1, in this research, as part of the HPC Midlands+ consortium.

\section{SUPPLEMENTARY MATERIAL}

The Supplementary Material for this article can be found online at: https://www.frontiersin.org/articles/10.3389/fchem. 2020.574038/full\#supplementary-material

Downs, C. A., Kramarsky-Winter, E., Fauth, J. E., Segal, R., Bronstein, O., Jeger, R., et al. (2013). Toxicological effects of the sunscreen UV filter, benzophenone-2, on planulae and in vitro cells of the coral, stylophora pistillata. Ecotoxicology 23, 175-191. doi: 10.1007/s10646-013-1161-y

Downs, C. A., Kramarsky-Winter, E., Segal, R., Fauth, J., Knutson, S., Bronstein, O., et al. (2016). Toxicopathological effects of the sunscreen UV filter, oxybenzone (benzophenone-3), on coral planulae and cultured primary cells and its environmental contamination in Hawaii and the U.S. virgin Islands. Arch. Environ. Contam. Toxicol. 70, 265-288. doi: 10.1007/s00244-015-0227-7

Eller, M. S., and Gilchrest, B. A. (2000). Tanning as part of the eukaryotic SOS response. Pigment Cell Res. 13, 94-97. doi: 10.1034/j.1600-0749.13.s8.17.x

Gao, Q., and Garcia-Pichel, F. (2011). Microbial ultraviolet sunscreens. Nat. Rev. Microbiol. 9, 791-802. doi: 10.1038/nrmicro2649

Grieco, C., Kohl, F. R., Zhang, Y., Natarajan, S., Blancafort, L., and Kohler, B. (2018). Intermolecular hydrogen bonding modulates o-h photodissociation in molecular aggregates of a catechol derivative. Photochem. Photobiol. 95, 163-175. doi: 10.1111/php.13035

Grubb, M. P., Orr-Ewing, A. J., and Ashfold, M. N. R. (2014). KOALA: a program for the processing and decomposition of transient spectra. Rev. Sci. Instrum. 85:064104. doi: 10.1063/1.4884516

Hamm, P. (1995). Coherent effects in femtosecond infrared spectroscopy. Chem. Phys. 200, 415-429. doi: 10.1016/0301-0104(95)00262-6

Hatakeyama, M., Koizumi, K., Boero, M., Nobusada, K., Hori, H., Misonou, T., et al. (2019). Unique structural relaxations and molecular conformations of porphyra-334 at the excited state. J. Phys. Chem. B 123, 7649-7656. doi: $10.1021 /$ acs.jpcb.9b03744

Holick, M. F. (2004). Sunlight and vitamin D for bone health and prevention of autoimmune diseases, cancers, and cardiovascular disease. Am. J. Clin. Nutr. 80, 1678s-1688s. doi: 10.1093/ajcn/80.6.1678S

Holm, A.-K., Rini, M., Nibbering, E. T. J., and Fidder, H. (2003). Femtosecond $\mathrm{UV} / \mathrm{mid}$-IR study of photochromism of the spiropyran $1^{\prime}, 3^{\prime}$-dihydro- $1^{\prime}, 3^{\prime}, 3^{\prime}$ trimethyl-6-nitrospiro[2H-1-benzopyran-2,2 - $(2 \mathrm{H})$-indole] in solution. Chem. Phys. Lett. 376, 214-219. doi: 10.1016/S0009-2614(03)00949-7

Horbury, M. D., Flourat, A. L., Greenough, S. E., Allais, F., and Stavros, V. G. (2018). Investigating isomer specific photoprotection in a model plant sunscreen. Chem Comm 54, 936-939. doi: 10.1039/C7CC09061G

Horbury, M. D., Holt, E. L., Mouterde, L. M. M., Balaguer, P., Cebrián, J., Blasco, L., et al. (2019). Towards symmetry driven and nature inspired UV filter design. Nat. Commun. 10:4748. doi: 10.1038/s41467-019-12719-Z

Horbury, M. D., Quan, W.-D., Flourat, A. L., Allais, F., and Stavros, V. G. (2017). Elucidating nuclear motions in a plant sunscreen during photoisomerization through solvent viscosity effects. Phys. Chem. Chem. Phys. 19, 21127-21131. doi: 10.1039/C7CP04070A

Humble, M. B. (2010). Vitamin D, light and mental health. J. Photochem. Photobiol. B Biol. 101, 142-149. doi: 10.1016/j.jphotobiol.2010.08.003

Klamt, A., and Schüürmann, G. (1993). COSMO: a new approach to dielectric screening in solvents with explicit expressions for the screening energy and its gradient. J. Chem. Soc. Perkin. Trans. 2, 799-805. doi: 10.1039/P299300 00799

Koizumi, K., Hatakeyama, M., Boero, M., Nobusada, K., Hori, H., Misonou, T., et al. (2017). How seaweeds release the excess energy from sunlight to surrounding sea water. Phys. Chem. Chem. Phys. 19, 15745-15753. doi: 10.1039/C7CP02699D 
Kollias, N., Sayre, R. M., Zeise, L., and Chedekel, M. R. (1991). New trends in photobiology; photoprotection by melanin. J. Photochem. Photobiol. B 9, 135-160. doi: 10.1016/1011-1344(91)80147-A

Losantos, R., Churio, M. S., and Sampedro, D. (2015). Computational exploration of the photoprotective potential of gadusol. ChemistryOpen 4, 155-160. doi: 10.1002/open.201402125

Losantos, R., Funes-Ardoiz, I., Aguilera, J., Herrera-Ceballos, E., Garcia-Iriepa, C., Campos, P. J., et al. (2017). Rational design and synthesis of efficient sunscreens to boost the solar protection factor. Angew. Chem. Int. Ed. 56, 2632-2635. doi: 10.1002/anie.201611627

Losantos, R., Lamas, I., Montero, R., Longarte, A., and Sampedro, D. (2019). Photophysical characterization of new and efficient synthetic sunscreens. Phys. Chem. Chem. Phys. 21, 11376-11384. doi: 10.1039/C9CP01267B

Lucas, R., McMichael, T., Smith, W., and Armstrong, B. (2006). "Solar ultraviolet radiation. global burden of disease from solar ultraviolet radiation," in Environmental Burden of Disease Series, No. 13 eds. A. Prüss-Üstün, H. Zeeb, C. Mathers, and M. Repacholi (Geneva: World Health Organization), 1-87.

Luo, J., Liu, Y., Yang, S., Flourat, A. L., Allais, F., and Han, K. (2017). Ultrafast barrierless photoisomerization and strong ultraviolet absorption of photoproducts in plant sunscreens. J. Phys. Chem. Lett. 8, 1025-1030. doi: 10.1021 acs.jpclett.7b00083

Moliné, M., Arbeloa, E. M., Flores, M. R., Libkind, D., Farías, M. E., Bertolotti, S. G., et al. (2011). UVB photoprotective role of mycosporines in yeast: photostability and antioxidant activity of mycosporine-glutaminol-glucoside. Radiat. Res. 175, 44-50. doi: 10.1667/RR2245.1

Mullen, K. M., and Van Stokkum, I. H. M. (2007). TIMP: an R package for modeling multi-way spectroscopic measurements. J. Stat. Softw. 18, 1-46. doi: $10.18637 /$ jss.v018.i03

Murdock, D., Harris, S. J., Clark, I. P., Greetham, G. M., Towrie, M., Orr-Ewing, A. J., et al. (2015). UV-induced isomerization dynamics of N-Methyl-2-pyridone in solution. J. Phys. Chem. A 119, 88-94. doi: 10.1021/jp511818k

Murdock, D., Harris, S. J., Luke, J., Grubb, M. P., Orr-Ewing, A. J., and Ashfold, M. N. R. (2014). Transient UV pump-IR probe investigation of heterocyclic ring-opening dynamics in the solution phase: the role played by $n \sigma^{*}$ states in the photoinduced reactions of thiophenone and furanone. Phys. Chem. Chem. Phys. 16, 21271-21279. doi: 10.1039/C4CP03653K

Nibbering, E. T. J., Fidder, H., and Pines, E. (2005). ULTRAFAST CHEMISTRY: using time-resolved vibrational spectroscopy for interrogation of structural dynamics. Annu. Rev. Phys. Chem. 56, 337-367. doi: 10.1146/annurev.physchem.56.092503.141314

Perdew, J. P., Ernzerhof, M., and Burke, K. (1996). Rationale for mixing exact exchange with density functional approximations. J. Chem. Phys. 105, 9982-9985. doi: 10.1063/1.472933

Rastogi, R. P., and Incharoensakdi, A. (2014). UV radiation-induced biosynthesis, stability and antioxidant activity of mycosporine-like amino acids (MAAs) in a unicellular cyanobacterium gloeocapsa sp. CU2556. J. Photochem. Photobiol. B Biol. 130, 287-292. doi: 10.1016/j.jphotobiol.2013.12.001

Rini, M., Holm, A.-K., Nibbering, E. T. J., and Fidder, H. (2003). Ultrafast UV-midIR investigation of the ring opening reaction of a photochromic spiropyran. J. Am. Chem. Soc. 125, 3028-3034. doi: 10.1021/ja028262j

Sampedro, D. (2011). Computational exploration of natural sunscreens. Phys. Chem. Chem. Phys. 13, 5584-5586. doi: 10.1039/c0cp02901g

Schaap, I., and Slijkerman, D. M. E. (2018). An environmental risk assessment of three organic UV-filters at lac bay, bonaire, southern caribbean. Mar. Pollut. Bull. 135, 490-495. doi: 10.1016/j.marpolbul.2018.07.054

Sinha, R. P., Klisch, M., Gröniger, A., and Häder, D.-P. (2000). Mycosporine-like amino acids in the marine red alga gracilaria cornea - effects of UV and heat. Environ. Exp. Bot. 43, 33-43. doi: 10.1016/S0098-8472(99)00043-X

Sinha, R. P., Singh, S. P., and Hader, D. P. (2007). Database on mycosporines and mycosporine-like amino acids (MAAs) in fungi, cyanobacteria, macroalgae, phytoplankton and animals. J. Photochem. Photobiol. B Biol. 89, 29-35. doi: 10.1016/j.jphotobiol.2007.07.006
Snellenburg, J. J., Laptenok, S., Seger, R., Mullen, K. M., and Van Stokkum, I. H. M. (2012). Glotaran: a java-based graphical user interface for the R package TIMP. J. Stat. Softw. 49, 1-22. doi: 10.18637/jss.v049.103

Sui, X.-X., Li, L., Zhao, Y., Wang, H.-G., Pei, K.-M., and Zheng, X. (2012). Resonance raman and density functional study of the excited state structural dynamics of 3-amino-2-cyclohexen-1-one in water and acetonitrile solvents. Spectrochim. Acta A 85, 165-172. doi: 10.1016/j.saa.2011.09.055

Turner, M. A. P., Turner, R. J., Horbury, M. D., Hine, N. D. M., and Stavros, V. G. (2019). Examining solvent effects on the ultrafast dynamics of catechol. J. Chem. Phys. 151:084305. doi: 10.1063/1.5116312

Valiev, M., Bylaska, E. J., Govind, N., Kowalski, K., Straatsma, T. P., Van Dam, H. J. J., et al. (2010). NWChem: a comprehensive and scalable open-source solution for large scale molecular simulations. Comput. Phys. Commun. 181, 1477-1489. doi: 10.1016/j.cpc.2010.04.018

Wang, S. Q., and Lim, H. W. (2016). Principles and Practice of Photoprotection. Switzerland: ADIS.

Warshaw, E. M., Wang, M. Z., Maibach, H. I., Belsito, D. V., Zug, K. A., Taylor, J. S., et al. (2013). Patch test reactions associated with sunscreen products and the importance of testing to an expanded series: retrospective analysis of North American contact dermatitis group data, 2001 to 2010. Dermatitis 24, 176-182. doi: 10.1097/DER.0b013e3182983845

White, J. D., Cammack, J. H., and Sakuma, K. (1989). The synthesis and absolute configuration of mycosporins. A novel application of the Staudinger reaction. J. Am. Chem. Soc. 111, 8970-8972. doi: 10.1021/ja00206a059

White, J. D., Cammack, J. H., Sakuma, K., Rewcastle, G. W., and Widener, R. K. (1995). Transformations of quinic acid. Asymmetric synthesis and absolute configuration of mycosporin i and mycosporin-gly. J. Org. Chem. 60, 3600-3611. doi: 10.1021/jo00117a008

Winget, P., Dolney, D. M., Giesen, D. J., Cramer, C. J., and Truhlar, D. G. (1999). Minnesota Solvent Descriptor Database. Minneapolis, MN: Department of Chemistry and Supercomputer Institute; University of Minnesota.

Woolley, J. M., Staniforth, M., Horbury, M. D., Richings, G. W., Wills, M., and Stavros, V. G. (2018). Unravelling the photoprotection properties of mycosporine amino acid motifs. J. Phys. Chem. Lett. 9, 3043-3048. doi: 10.1021/acs.jpclett.8b00921

York, D. M., and Karplus, M. (1999). A smooth solvation potential based on the conductor-like screening model. J. Phys. Chem. A 103, 11060-11079. doi: 10.1021/jp9920971

Zhao, X., Luo, J., Liu, Y., Pandey, P., Yang, S., Wei, D., et al. (2019a). Substitution dependent ultrafast ultraviolet energy dissipation mechanisms of plant sunscreens. J. Phys. Chem. Lett. 10, 5244-5249. doi: 10.1021/acs.jpclett.9b02175

Zhao, X., Luo, J., Yang, S., and Han, K. (2019b). New insight into the photoprotection mechanism of plant sunscreens: adiabatic relaxation competing with nonadiabatic relaxation in the cis $\rightarrow$ trans photoisomerization of methyl sinapate. J. Phys. Chem. Lett. 10, 4197-4202. doi: 10.1021/acs.jpclett.9b01651

Zuehlsdorff, T. J., Haynes, P. D., Payne, M. C., and Hine, N. D. M. (2017) Predicting solvatochromic shifts and colours of a solvated organic dye: The example of nile red. J. Chem. Phys. 146:124504. doi: 10.1063/1.49 79196

Conflict of Interest: The authors declare that the research was conducted in the absence of any commercial or financial relationships that could be construed as a potential conflict of interest.

Copyright $\odot 2020$ Whittock, Turner, Coxon, Woolley, Horbury and Stavros. This is an open-access article distributed under the terms of the Creative Commons Attribution License (CC BY). The use, distribution or reproduction in other forums is permitted, provided the original author(s) and the copyright owner(s) are credited and that the original publication in this journal is cited, in accordance with accepted academic practice. No use, distribution or reproduction is permitted which does not comply with these terms. 